\title{
The art of selective killing: plasmid toxin/antitoxin systems and their technological applications
}

\author{
Daniel Stieber*, Philippe Gabant, and Cédric Y. Szpirer \\ Delphi Genetics SA, Rue Clément Ader 16, Gosselies, Belgium, www.delphigenetics.com \\ *dstieber@delphigenetics.com
}

\section{INTRODUCTION}

Technologies using recombinant DNA have become of paramount importance in most biology laboratories today and plasmid toxin/antitoxin (T/A) systems have proven to be very useful by facilitating different key technologies of molecular cloning. Several applications and the underlying technologies are highlighted in this review.

\section{PRINCIPLES OF THE T/A TECHNOLOGY}

Most bacterial strains harbor plasmids that are maintained with remarkable stability. A large variety of plasmids encode systems that act when other control mechanisms have failed, i.e., when plasmid-free progeny is generated during replication. The mechanisms that control plasmid maintenance by T/A loci are well known: the antagonistic regulators that neutralize the toxins are metabolically unstable. Rapid depletion of these unstable regulators occurs in newborn, plasmid-free cells. As the same cells have inherited stable toxin molecules from the mother cell, the toxin will no longer be neutralized by the antitoxin, leading to the killing of the plasmid-free cells. This mechanism effectively reduces the proliferation of plasmid-free cells in growing bacterial populations (1).

The most widely studied T/A system so far is the ccd system located on the $\mathrm{F}$ plasmid (2). The ccd system is composed of two genes, $c c d A$ and $c c d B$, encoding small proteins: the $\mathrm{Ccd} A$ antidote $(8.7 \mathrm{kDa})$ and the $\mathrm{CcdB}$ toxin $(11.7 \mathrm{kDa})$. The CcdB protein acts as a poison because it selectively targets the Escherichia coli DNA gyrase, a bacterial topoisomerase II. Early studies of this T/A system were performed at the Université Libre de Bruxelles (ULB). Today new applications are commercialized by Delphi Genetics SA, a spin off company of the ULB founded by the researchers who developed the use of T/A systems as selectable markers.

\section{POSITIVE SELECTION VECTORS}

One of the major drawbacks in DNA cloning is the scarcity of the insertion event of the DNA insert into the plasmid. Typically, less than $10 \%$ of the vectors circularize with an insert. The $90 \%$ of "empty" vectors represent a major background in molecular cloning experiments. From the early development of molecular cloning, identifying vectors with an insert has been a frustrating and timeconsuming step for the investigator. The development of vectors employing T/A systems circumvents these issues and permits growth of bacteria harboring insert-bearing plasmids only. Typically, the vectors used in these systems express a gene product that is lethal to certain bacterial hosts. The lethal gene is inactivated by insertion of a segment of foreign DNA and therefore, toxicity is relieved. The most efficient technical solution remains the killing of bacteria harboring an insertless vector, or the selection of bacteria harboring the recombinant vector, the so-called positive selection. T/A systems are of paramount technological significance for positive selection vectors. The ccdB technology (3), pioneered by Researchers at ULB and commercially developed by Delphi Genetics, has proven to be a successful approach for constructing positive selection vectors. The flexibility of the $\mathrm{ccdB}$ selection was proven by the system of vectors for different cloning applications based on Delphi Genetics' ccdB technology sold by the licensee of this technology, Invitrogen Inc. CcdB-based positive selection vectors featuring different copy numbers, a broad-host range and/or transfer properties were developed to facilitate cloning for molecular genetics. The large portfolio of positive selection vectors sold by Invitrogen has nowadays become a standard tool in any normal molecular biology laboratory.

Another system based on the ccdB technology is the Gateway system, also commercialized by Invitrogen. In this system, site-specific recombination is used to insert a gene of interest into the vector, and the recombinants are 
selected by the replacement of the $c c d B$ gene by the gene of interest. This powerful method allows rapid and efficient transfer of all the genes from an organism from one vector to different vectors (i.e., expression vectors) by automatic sub-cloning.

The next generation of positive selection vectors has recently been introduced by Delphi Genetics. These new versatile vectors are based on a novel application of the T/A properties of the ccd proteins; The StabyCloning ${ }^{\mathrm{TM}}$ system from Delphi Genetics uses both the ccdA and the $\mathrm{ccdB}$ proteins. The bacteria used in this system contain the $c c d B$ gene in their chromosome. A truncated inactive version of the antitoxin $(c c d A)$ gene is present in the linearized plasmid vector. When a sequence of 14 base pairs is added to the $5^{\prime}$-end of the DNA fragment to be cloned, the fusion of this sequence with the truncated gene restores an active antitoxin protein able to counteract the action of the toxin. The 14-bp sequence is incorporated to the DNA fragment using one modified PCR primer. This system allows for the positive selection of recombinant plasmids only and for the selection of the correct orientation of the cloned fragment in the vector (Figure 1). An additional advantage of this procedure is the speed of the whole procedure: 1 hour until plating.

Due to the innovative positive selection technique the background is virtually nil and the use of antibiotics and the associated pitfalls (e.g., satellite colonies when selecting on Ampiciline) is avoided. Another important feature of this technology is that all recombinants are independent clones; which allows for direct plating, furthermore

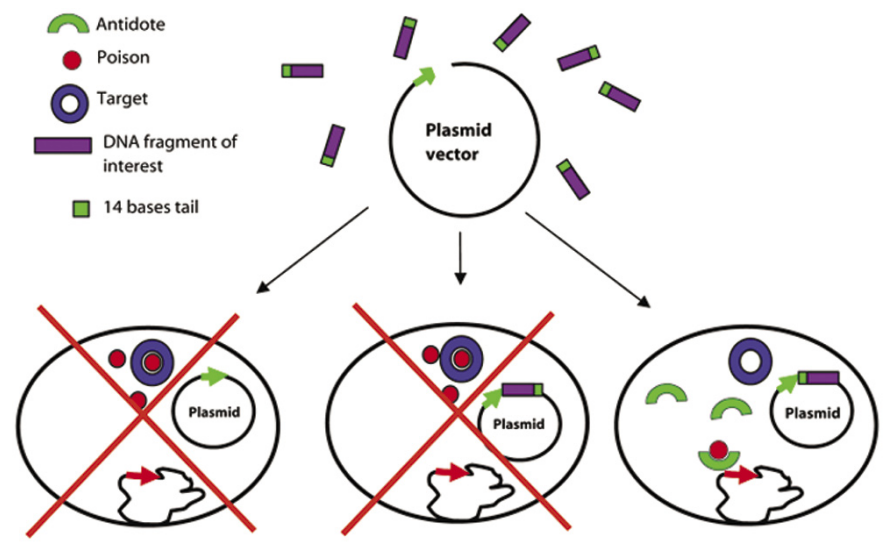

Figure 1. Principle of the StabyCloning ${ }^{\mathrm{TM}}$ system the system is usable in any culture medium. This cloning method has recently been shown to be very efficient for the generation of microsatellite libraries (4).

\section{PLASMID STABILIZATION SYSTEMS FOR THE PRODUCTION OF PROTEINS}

Plasmid instability is a significant concern in the utilization of microorganisms for protein or DNA production. Usually, these processes require the use of a bacterial plasmid construct as a vector carrying a gene to be expressed. In a fermentation process, cells losing the plasmidic construct exhibit a higher fitness than construct-free cells, and the former rapidly overcome the later in the bacterial population. Antibiotic resistance genes are the most commonly used selectable markers in fermentation procedures to avoid plasmid-free cells to survive and dominate the culture. However several important drawbacks, such as high cost and regulatory issues, are associated with the use of antibiotics. Alternative plasmid-stabilization strategies have been developed. The most efficient strategy is to use toxin/antitoxin genes that induce host killing upon plasmid loss. In previously developed stabilization systems using toxin/antitoxin genes (Hok-Sok and parDE T/A systems), the sequence encoding the full operon is cloned into the plasmid to be stabilized. In plasmid-cured bacteria, de novo synthesis of the T/A pair ends, and as a result of antitoxin degradation, the poison is free to exert its toxicity, eventually causing cell death. Unfortunately, these toxin/antitoxin systems can only delay, but not prevent, the takeover of a culture by plasmidfree cells. Indeed, after plasmid loss, dilution of the poison by bacterial growth before complete antidote degradation can allow cells to survive and produce a high-fitness line of plasmid-free cells.

The StabyExpress ${ }^{\mathrm{TM}}$ technology developed by researchers at Delphi Genetics is a new and highly effective stabilization system based on the use of the $c c d$ toxin/antitoxin genes that addresses these pitfalls. The antitoxin gene has been separated from the poison gene, localizing the former in the plasmid and integrating the later in the bacterial chromosome. The antitoxin gene in the plasmid is under the control of a constitutive promoter whereas the expression of the toxin gene in the chromosome is under the control of a promoter strongly repressed by the antitoxin protein. Thus when the antitoxin is present in the bacteria, the toxin is not produced, whereas upon plasmid loss the toxin is induced causing cell death. This separated-component-stabilization (SCS) (5) strategy: ( $i$ ) allows for perfect stabilization without the use 
of antibiotics during the production process; (ii) increases three to five times the recombinant protein production levels; and (iii) does not require any specific modification of the DNA or protein production process or culture medium.

To demonstrate the efficiency of this technology, we tested the system in E. coli. The results show a perfect plasmid stabilization of several vectors unstable without our technology. Figure 2 illustrates the comparison of the production of a $69 \mathrm{kda}$ protein with and without the StabyExpress ${ }^{\mathrm{TM}}$ technology.

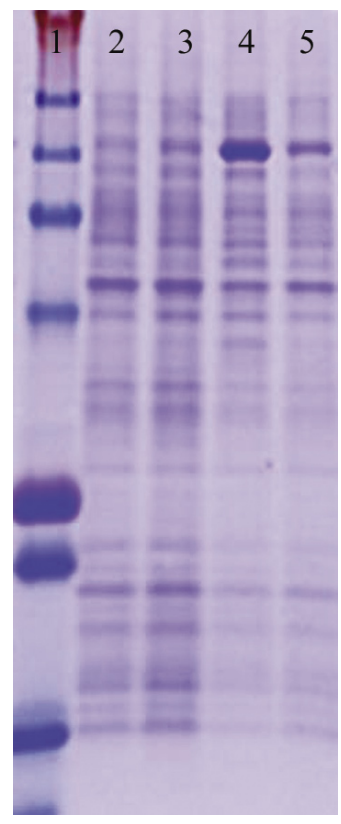

Figure 2

Using StabyExpress ${ }^{\mathrm{TM}}$ the plasmid is perfectly stable before and after the induction period. On the contrary, using a conventional BL21(DE3) strain, the plasmid is not stably maintained. Consequently, the production of the protein of interest is higher ( 3 to 5 times) with StabyExpress ${ }^{\mathrm{TM}}$ (Lane 4) than using a conventional expression system with antibiotics (Lane 5). Lane 1 is a size marker, and Lane 2 and 3 are the uninduced controls for the StabyExpress ${ }^{\mathrm{TM}}$ System and the classical system respectively.

Regarding protein expression, Delphi Genetics has developed several new tools based on the proven Staby ${ }^{\mathrm{TM}}$ technology: StabyCodon ${ }^{\mathrm{TM}}$ addressing the problem of codon usage adaptation in $E$. coli for the efficient expression of proteins that contain rare codons and the Cherry ${ }^{\mathrm{TM}}$ range of products, which allow for the direct visualization and the accurate quantification of the protein of interest by fusing the POI to a red-colored, highly soluble reporter Tag.

\section{CONCLUSION}

The potent bacterial toxins of T/A systems have been fine-tuned by natural selection so that mutants resistant to them arise at very low frequency. Recent bioinformatic studies of bacterial genomes revealed the presence of hundreds of these systems in the chromosomes (6). This demonstrated diversity opens the door for applications in other hosts than the laboratory E. coli strains. Each toxin has evolved in parallel with a specific antitoxin enabling cells to carry and express the toxin gene and yet survive under suitable conditions. This mechanism for controlling cell survival offers biotechnologists a molecular toolbox for selecting recombinants and stabilizing plasmids. The major advantages of T/A systems are: ( $i$ ) their small size (about 100 amino acids for the poison and 90 amino acids for the antidote); (ii) the efficiency of the toxin selected to exert its activity in bacteria (resistant mutants are rare or nonexistent); and (iii) their broad range of use. Some T/A systems are active in several Gram-negative bacteria or even in yeast and mammalian cells. The stabilization system described here should thus be adaptable to other microorganisms or eukaryotic cells.

\section{REFERENCES}

1. Hayes F. (2003), Science 301(5639):1496-9.

2. Van Melderen L. (2002), Int. J. Med. Microbiol. 291(6-7):537-44.

3. Bernard et al. (1994), Gene 148(1):71-4.

4. Tzika et al. (2008), Molecular Ecology 17(2): 533-44.

5. Szpirer et al. (2005), BioTechniques 38(5):775-81.

6. Pandey et al. (2005), NAR 33(3):966-76. 